\title{
ISOTROPY OF 8-DIMENSIONAL QUADRATIC FORMS OVER FUNCTION FIELDS OF QUADRICS
}

\author{
OLEG T. IZHBOLDIN AND NIKITA A. KARPENKO
}

\begin{abstract}
Let $F$ be a field of characteristic different from 2 and $\phi$ be an anisotropic 8-dimensional quadratic form over $F$ with trivial determinant. We study the last open cases in the problem of describing the quadratic forms $\psi$ such that $\phi$ becomes isotropic over the function field $F(\psi)$.
\end{abstract}

\section{INTRODUCTION}

Let $F$ be a field of characteristic different from 2 and let $\phi$ and $\psi$ be two anisotropic quadratic forms over $F$. An important problem in the algebraic theory of quadratic forms is to find conditions on $\phi$ and $\psi$ so that $\phi_{F(\psi)}$ is isotropic. More precisely, one studies the question whether the isotropy of $\phi$ over $F(\psi)$ is standard in a sense.

In this paper we consider the case where $\phi$ is an 8-dimensional anisotropic quadratic form with trivial determinant. Necessity of certain sufficient conditions for isotropy of $\phi$ over $F(\psi)$ was studied by A. Laghribi; we call the isotropy, caused by one of these conditions, L-standard:

Definition. Let $\phi$ and $\psi$ be anisotropic quadratic forms such that $\phi_{F(\psi)}$ is isotropic. Besides we suppose that $\operatorname{dim} \phi=8$ and $\operatorname{det} \phi=1$. We say that the isotropy of $\phi_{F(\psi)}$ is L-standard, if at least one of the following conditions holds:

- there exists a half-neighbor $\phi^{*}$ of $\phi$ such that $\psi \subset \phi^{*}$;

- there exists a 5-dimensional subform $\phi_{0} \subset \phi$ with the following two properties:

- the form $\phi_{0}$ is a Pfister neighbor,

- the form $\left(\phi_{0}\right)_{F(\psi)}$ is isotropic.

Otherwise, we say that the isotropy is non-L-standard.

In the case when $\operatorname{dim} \psi \geq 5$, the isotropy of $\phi_{F(\psi)}$ is always L-standard ([18], [17], see also [8]). The main result of our paper is the following

Theorem. Let $\phi$ be an anisotropic 8-dimensional quadratic form with $\operatorname{det} \phi=$ 1 and $\psi$ be a 4-dimensional quadratic form with $\operatorname{det} \psi \neq 1$. Suppose that $\phi_{F(\psi)}$ is isotropic. Then the isotropy of $\phi_{F(\psi)}$ is L-standard except (possibly) the case ind $C(\phi)=\operatorname{ind}\left(C(\phi) \otimes_{F} C_{0}(\psi)\right)=4$.

For the case where $\operatorname{dim} \psi=3$ or where $\operatorname{dim} \psi=4$ and $\operatorname{det} \psi=1$ see $\S 8$.

Date: August, 1997. 


\section{TeRminology And NOtATiOn}

1.1. Quadratic forms. Mainly, we use notation of [19] and [27]. However there is certain slight difference: we denote by $\left\langle\left\langle a_{1}, \ldots, a_{n}\right\rangle\right\rangle$ the $n$-fold Pfister form $\left\langle 1,-a_{1}\right\rangle \otimes \cdots \otimes\left\langle 1,-a_{n}\right\rangle$. We denote by $P_{n}(F)$ the set of all $n$-fold Pfister forms; $G P_{n}(F)$ is the set of the forms similar to a form from $P_{n}(F)$. For any field extension $L / F$, we denote by $P_{n}(L / F)$ the set of forms from $P_{n}(F)$ hyperbolic over $L ; G P_{n}(L / F)$ is the set of the forms similar to a form from $P_{n}(L / F)$.

We recall that a quadratic form $\psi$ is called a (Pfister) neighbor (of a Pfister form $\pi$ ), if it is similar to a subform in $\pi$ and $\operatorname{dim} \phi>\frac{1}{2} \operatorname{dim} \pi$. Two quadratic forms $\phi$ and $\phi^{*}$ are half-neighbors, if $\operatorname{dim} \phi=\operatorname{dim} \phi^{*}$ and there exists $s \in F^{*}$ such that the sum $\phi \perp s \phi^{*}$ is similar to a Pfister form.

For a quadratic form $\phi$ of dimension $\geq 3$, we denote by $X_{\phi}$ the projective variety given by the equation $\phi=0$. We set $F(\phi)=F\left(X_{\phi}\right)$ if $\operatorname{dim} \phi \geq 3$; $F(\phi)=F(\sqrt{d})$ if $\operatorname{dim} \phi=2$ and $d=d_{ \pm} \phi \neq 1$; and $F(\phi)=F$ otherwise.

1.2. Algebras. We consider only finite-dimensional $F$-algebras.

For a central simple $F$-algebra $D$, we denote by $\operatorname{deg}(D),[D]$, and $\exp (D)$ respectively the degree of $D$, the class of $D$ in the Brauer group $\operatorname{Br}(F)$, and the exponent of $D$, i.e., the order of $[D]$ in the Brauer group.

For a simple $F$-algebra $A$, we denote by ind $(A)$ the Schur index of $A$. For an algebra $B$ of the form $B=A \times \cdots \times A$ with simple $A$, we set ind $B=\operatorname{ind} A$.

Let $\phi$ be a quadratic form. We denote by $C(\phi)$ the Clifford algebra of $\phi$. By $C_{0}(\phi)$ we denote the even part of $C(\phi)$. Note that for any quadratic $F$-form $\psi$ and any central simple $F$-algebra $D$, the index of $C_{0}(\psi) \otimes_{F} D$ is well-defined.

If $\phi \in I^{2}(F)$ then $C(\phi)$ is a central simple algebra. Its class $[C(\phi)]$ in the Brauer group $\mathrm{Br}_{2}(F)$ is denoted by $c(\phi)$.

Let $D$ be a central simple algebra. We denote by $X_{D}$ the Severi-Brauer variety of $D$ and by $F(D)$ the function field $F\left(X_{D}\right)$. For another central simple $F$-algebra $D^{\prime}$ and for a quadratic $F$-form $\psi$ of dimension $\geq 3$, we set $F\left(D^{\prime}, D\right) \stackrel{\text { def }}{=} F\left(X_{D^{\prime}} \times X_{D}\right)$ and $F(\psi, D) \stackrel{\text { def }}{=} F\left(X_{\psi} \times X_{D}\right)$.

1.3. Cohomology groups. By $H^{*}(F)$ we denote the graded ring of Galois cohomology $H^{*}(F, \mathbb{Z} / 2 \mathbb{Z}) \stackrel{\text { def }}{=} H^{*}\left(\operatorname{Gal}\left(F_{\text {sep }} / F\right), \mathbb{Z} / 2 \mathbb{Z}\right)$.

For $n=0,1,2,3$, there is a homomorphism $e^{n}: I^{n}(F) \rightarrow H^{n}(F)$ which is uniquely determined by the condition $e^{n}\left(\left\langle\left\langle a_{1}, \ldots, a_{n}\right\rangle\right\rangle\right)=\left(a_{1}, \ldots, a_{n}\right)$ (see [1]). The homomorphism $e^{n}$ is surjective and $\operatorname{ker} e^{n}=I^{n+1}(F)$ for $n=0,1,2,3$ (see [20], [21], and [25]).

We also work with the cohomology groups $H^{n}(F, \mathbb{Q} / \mathbb{Z}(i)),(i=0,1,2)$, defined by B. Kahn (see [11]). For $n=1,2,3$, the group $H^{n}(F)$ is naturally identified with $\operatorname{Tors}_{2} H^{n}(F, \mathbb{Q} / \mathbb{Z}(n-1))$.

1.4. $K$-theory and Chow groups. For a smooth algebraic $F$-variety $X$, its Grothendieck ring is denoted by $K(X)$. This ring is supplied with the filtration by codimension of support (also called the topological filtration). 
We fix an algebraic closure $\bar{F}$ of the base field $F$ and denote by $\bar{X}$ the $\bar{F}$ variety $X_{\bar{F}}$. If the variety $X$ is projective homogeneous, we identify $K(X)$ with a subring of $K(\bar{X})$ via the restriction homomorphism which is injective by $[22]$.

For a ring (or a group) with filtration $A$, we denote by $\mathrm{G}^{*} A$ the adjoint graded ring (resp., the adjoint graded group).

There is a canonical surjective homomorphism of the graded Chow ring $\mathrm{CH}^{*}(X)$ onto $\mathrm{G}^{*} K(X)$, its kernel consists only of torsion elements and is trivial in the 0 -th, 1-st, and 2-nd graded components $([28, \S 9])$.

Let $X_{1}$ and $X_{2}$ be two smooth $F$-varieties. For any $x_{1} \in K\left(X_{1}\right)$ and $x_{2} \in$ $K\left(X_{2}\right)$, we denote by $x_{1} \otimes x_{2}$ the product $p r_{1}^{*}\left(x_{1}\right) \cdot p r_{2}^{*}\left(x_{2}\right) \in K\left(X_{1} \times X_{2}\right)$ where $p r_{1}^{*}$ and $p r_{2}^{*}$ are the pull-backs with respect to the projections $p r_{1}$ and $p r_{2}$ of $X_{1} \times X_{2}$ on $X_{1}$ and $X_{2}$ respectively. For an $\mathcal{O}_{X_{1}}$-module $\mathcal{F}_{1}$ and an $\mathcal{O}_{X_{2}}$-module $\mathcal{F}_{2}$, we denote by $\mathcal{F}_{1} \otimes \mathcal{F}_{2}$ the tensor product $\operatorname{pr}_{1}^{*}\left(\mathcal{F}_{1}\right) \otimes_{\mathcal{O}_{X}} \operatorname{pr}_{2}^{*}\left(\mathcal{F}_{2}\right)$.

1.5. Relative groups. Let $\Phi$ be an arbitrary functor on the category of fields (of characteristic $\neq 2$ ) with values in the category of abelian groups. For a field extension $L / F$ we use the notation $\Phi(L / F)$ for $\operatorname{ker}(\Phi(F) \rightarrow \Phi(L))$. Here is a list of examples that we need in this paper: $W(L / F), I^{n}(L / F), H^{n}(L / F)$, and $H^{n}(L / F, \mathbb{Q} / \mathbb{Z}(i))$.

2. The groups $H^{3}(F(\psi, D) / F)$ And $I^{3}(F(\psi, D) / F)$

Proposition 2.1. Let $D$ be a central simple F-algebra of exponent 2 and $\psi$ be a quadratic form of dimension $\geq 3$. Then there exists a natural isomorphism

$$
\frac{H^{3}(F(\psi, D) / F)}{H^{3}(F(\psi) / F)+H^{3}(F(D) / F)} \simeq \frac{\operatorname{Tors~}^{2} H^{2}\left(X_{\psi} \times X_{D}\right)}{p r_{\psi}^{*} \operatorname{Tors~}^{2} H^{2}\left(X_{\psi}\right)+p r_{D}^{*} \operatorname{Tors~}^{2}\left(X_{D}\right)}
$$

where $p r_{\psi}^{*}$ and $p r_{D}^{*}$ are the pull-backs with respect to the projection $p r_{\psi}$ and $p r_{D}$ of $X_{\psi} \times X_{D}$ to $X_{\psi}$ and $X_{D}$.

Proof. By [7, Prop. 2.2] the factor group

$$
\frac{H^{3}(F(\psi, D) / F, \mathbb{Q} / \mathbb{Z}(2))}{\left.H^{3}(F(\psi) / F, \mathbb{Q} / \mathbb{Z}(2))+H^{3}(F(D) / F), \mathbb{Q} / \mathbb{Z}(2)\right)}
$$

is isomorphic to

$$
\frac{\operatorname{Tors} \mathrm{CH}^{2}\left(X_{\psi} \times X_{D}\right)}{p r_{\psi}^{*} \text { Tors } \mathrm{CH}^{2}\left(X_{\psi}\right)+p r_{D}^{*} \text { Tors } \mathrm{CH}^{2}\left(X_{D}\right)} .
$$

Now it is sufficient to apply isomorphisms

$$
\begin{aligned}
H^{3}(F(\psi) / F, \mathbb{Q} / \mathbb{Z}(2)) & =H^{3}(F(\psi) / F), \\
H^{3}(F(D) / F, \mathbb{Q} / \mathbb{Z}(2)) & =H^{3}(F(D) / F), \\
H^{3}(F(\psi, D) / F, \mathbb{Q} / \mathbb{Z}(2)) & =H^{3}(F(\psi, D) / F) .
\end{aligned}
$$

(see e.g. [7, Lemma 2.8], [4, Lemma A.8], [8]). 
Corollary 2.2. Let $D$ be a biquaternion algebra and $\psi$ be a 4-dimensional quadratic form. Then there exists a natural isomorphism

$$
\frac{H^{3}(F(\psi, D) / F)}{H^{3}(F(\psi) / F)+H^{3}(F(D) / F)} \simeq \operatorname{Tors~}^{2} H^{2}\left(X_{\psi} \times X_{D}\right) .
$$

In particular, $2 \cdot \operatorname{Tors} \mathrm{CH}^{2}\left(X_{\psi} \times X_{D}\right)=0$.

Proof. Since $\operatorname{dim} \psi=4$, we have Tors $\mathrm{CH}^{2}\left(X_{\psi}\right)=0$ (see e.g. [12, Theorem 6.1] or $\left[7\right.$, Lemma 2.4]). By [14, Proposition 5.3], we have Tors $\mathrm{CH}^{2}\left(X_{D}\right)=0$. To complete the proof it is sufficient to apply Proposition 2.1.

Lemma 2.3. Let $\psi$ be a quadratic form of dimension $\geq 3$. Then

1. the $\operatorname{map} e^{3}: P_{3}(F(\psi) / F) \rightarrow H^{3}(F(\psi) / F)$ is surjective;

2. $I^{3}(F(\psi) / F)+I^{4}(F)=P_{3}(F(\psi) / F)+I^{4}(F)$.

Proof. 1. Really, we have to verify that the set $H^{3}(F(\psi) / F)$ consists of symbols. This fact is proved in [1, Satz 5.6].

2. Follows from Item 1 and from injectivity of $e^{3}: I^{3}(F) / I^{4}(F) \rightarrow H^{3}(F)$.

Lemma 2.4. Let $D$ be a biquaternion algebra and $q$ be an Albert form of $D$. Then

1. $H^{3}(F(D) / F)=[D] \cup H^{1}(F)$;

2. $I^{3}(F(D) / F)+I^{4}(F)=\left\{q\langle\langle s\rangle\rangle \mid s \in F^{*}\right\}+I^{4}(F)$;

3. the map $e^{3}: I^{3}(F(D) / F) \rightarrow H^{3}(F(D) / F)$ is surjective.

Proof. 1. See [23, Corollary 4.5].

2. Obviously $e^{3}(q\langle\langle s\rangle\rangle)=[D] \cup(s)$. Now, it is sufficient to apply Item 1 and injectivity of $e^{3}: I^{3}(F) / I^{4}(F) \rightarrow H^{3}(F)$.

3. Follows from Items 1 and 2 .

Lemma 2.5. Let $D$ be a biquaternion algebra and $\psi$ be a quadratic form of dimension $\geq 3$. Then the natural homomorphism

$$
\frac{I^{3}(F(\psi, D) / F)+I^{4}(F)}{I^{3}(F(\psi) / F)+I^{3}(F(D) / F)+I^{4}(F)} \rightarrow \frac{H^{3}(F(\psi, D) / F)}{H^{3}(F(\psi) / F)+H^{3}(F(D) / F)}
$$

is injective.

In particular, the condition $H^{3}(F(\psi, D) / F)=H^{3}(F(\psi) / F)+H^{3}(F(D) / F)$ implies that $I^{3}(F(\psi, D) / F) \subset I^{3}(F(\psi) / F)+I^{3}(F(D) / F)+I^{4}(F)$.

Proof. Obvious consequence of the following facts:

a) $I^{3}(F) / I^{4}(F) \rightarrow H^{3}(F)$ is injective;

b) $\left.I^{3}(F(\psi) / F)\right) \rightarrow H^{3}(F(\psi) / F)$ is surjective (Lemma 2.3 );

c) $\left.I^{3}(F(D) / F)\right) \rightarrow H^{3}(F(D) / F)$ is surjective (Lemma 2.4).

Corollary 2.6. Let $D$ be a biquaternion algebra and $\psi$ be a quadratic form of dimension $\geq 3$ such that Tors $\mathrm{CH}^{2}\left(X_{\psi} \times X_{D}\right)=0$. Then $I^{3}(F(\psi, D) / F) \subset$ $I^{3}(F(\psi) / F)+I^{3}(F(D) / F)+I^{4}(F)$.

Proof. Follows from Lemma 2.5 and Proposition 2.1. 


\section{The group $K\left(X_{\psi} \times X_{D}\right)$}

In this section, $\psi$ is a quadratic $F$-form of dimension $4, D$ is a biquaternion $F$-algebra.

Lemma 3.1. Consider the tensor product $K\left(X_{\psi}\right) \otimes_{\mathbb{Z}} K\left(X_{D}\right)$ together with the filtration induced by the topological filtrations on $K\left(X_{\psi}\right)$ and $K\left(X_{D}\right)$. The adjoint graded group $G^{*}\left(K\left(X_{\psi}\right) \otimes K\left(X_{D}\right)\right)$ is torsion-free.

Proof. The adjoint graded group $G^{*} K\left(X_{\psi}\right)$ is torsion-free (see e.g [12]). The adjoint graded group $G^{*} K\left(X_{D}\right)$ is torsion-free as well (see [13, Example]). We have a surjection

$$
G^{*} K\left(X_{\psi}\right) \otimes G^{*} K\left(X_{D}\right) \rightarrow G^{*}\left(K\left(X_{\psi}\right) \otimes K\left(X_{D}\right)\right) .
$$

The left-hand side term is a finitely generated torsion-free abelian group, i.e. a free abelian group of finite rank. This rank coincides with the rank of the right-hand side term. Therefore, the map is an isomorphism.

We consider the subgroup $K\left(X_{\psi}\right) \otimes K\left(X_{D}\right)$ of $K\left(X_{\psi} \times X_{D}\right)$ together with the filtration induced by the topological filtration on $K\left(X_{\psi} \times X_{D}\right)$.

Lemma 3.2. The homomorphism $K\left(X_{\psi}\right) \otimes K\left(X_{D}\right) \rightarrow K\left(X_{\psi}\right) \otimes K\left(X_{D}\right)$ is an isomorphism of groups with filtrations.

Proof. It is a homomorphism of groups respecting the filtrations. First of all let us check that it is an isomorphism of groups, regardless the filtrations. It is evidently an epimorphism. So, we only have to check the injectivity. Since for any extension of the base field $F$, the restriction homomorphism on the product $K\left(X_{\psi}\right) \otimes K\left(X_{D}\right)$ is injective, it suffices to check the injectivity in a split situation. However, if $D$ splits, then $X_{D}$ is isomorphic to a projective space; therefore the map $K\left(X_{\psi}\right) \otimes K\left(X_{D}\right) \rightarrow K\left(X_{\psi}\right) \otimes K\left(X_{D}\right)$ is an isomorphism.

To finish the proof, it is suffices to show that the homomorphism of the adjoint graded groups is injective. Consider the commutative diagram

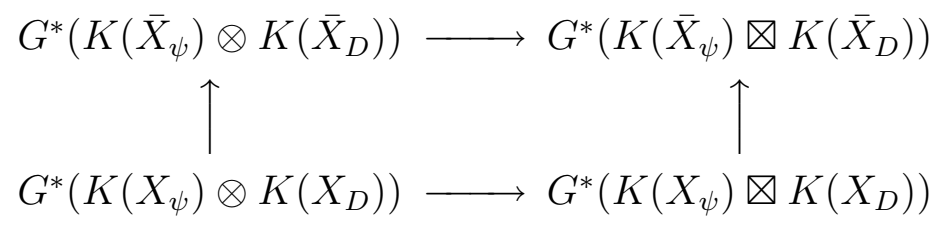

The left hand side arrow is injective since the group $G^{*}\left(K\left(X_{\psi}\right) \otimes K\left(X_{D}\right)\right)$ is torsion-free (Lemma 3.1) The upper arrow is injective because $\bar{X}_{D}$ is isomorphic to a projective space. Therefore the bottom arrow is injective too.

Corollary 3.3. The group $G^{*}\left(K\left(X_{\psi}\right) \otimes K\left(X_{D}\right)\right)$ is torsion-free.

We denote by $C$ the even Clifford algebra $C_{0}(\psi)$. Let $\mathcal{U}(2)$ be the Swan's sheaf $\mathcal{U}$ on the quadric $X_{\psi}([29, \S 6])$, twisted twice. It has a structure of $C$ module. Let $\mathcal{J}$ be the canonical sheaf on the Severi-Brauer variety $X_{D}$ ([24, $\S 8.4])$. It has a structure of $D$-module. 
Put $\mathcal{F} \stackrel{\text { def }}{=} \mathcal{U}(2) \otimes \mathcal{J}$. It is a sheaf on $X_{\psi} \times X_{D}$ with a structure of $C \otimes_{F} D$ module. Denote by $f$ the homomorphism $K(C \otimes D) \rightarrow K\left(X_{\psi} \times X_{D}\right)$ given by the functor of tensor multiplication by $\mathcal{F}$ over $C \otimes D$.

Put $\mathcal{G} \stackrel{\text { def }}{=} \mathcal{U}(2) \otimes \mathcal{J}^{\otimes 3}$. It is a sheaf on $X_{\psi} \times X_{D}$ with a structure of $C \otimes_{F} D^{\otimes 3}$ module. Consider the homomorphism $K\left(C \otimes D^{\otimes 3}\right) \rightarrow K\left(X_{\psi} \times X_{D}\right)$ given by the functor of tensor multiplication by $\mathcal{G}$ over $C \otimes D^{\otimes 3}$. Since the algebra $D^{\otimes 2}$ is split, the group $K\left(C \otimes D^{\otimes 3}\right)$ is canonically isomorphic (via Morita equivalence) to $K(C \otimes D)$. Denote by $g$ the the composition $K(C \otimes D) \stackrel{\sim}{\rightarrow}$ $K\left(C \otimes D^{\otimes 3}\right) \rightarrow K\left(X_{\psi} \times X_{D}\right)$.

Lemma 3.4. 1. The homomorphism

$$
K(C \otimes D)^{\oplus 2} \rightarrow K\left(X_{\psi} \times X_{D}\right) /\left(K\left(X_{\psi}\right) \otimes K\left(X_{D}\right)\right),
$$

induced by the homomorphisms $f+g$, is surjective.

2. If $C \otimes D$ is a skewfield, then $K\left(X_{\psi} \times X_{D}\right)=K\left(X_{\psi}\right) \otimes K\left(X_{D}\right)$.

Proof. 1. Using the Swan's computation of the K-theory for quadrics [29, Theorem 9.1] (with $\mathcal{U}(2)$ instead of $\mathcal{U}$ ) and a generalized Peyre's version [23, Proposition 3.1] of the Quillen's computation of K-theory for Severi-Brauer schemes [24, Theorem 4.1 of $\S 8]$, we get an isomorphism

$$
K(F)^{\oplus 4} \oplus K(C)^{\oplus 2} \oplus K(D)^{\oplus 4} \oplus K(C \otimes D)^{\oplus 2} \simeq K\left(X_{\psi} \times X_{D}\right)
$$

such that the image of $K(F)^{\oplus 4} \oplus K(C)^{\oplus 2} \oplus K(D)^{\oplus 4}$ is contained in $K\left(X_{\psi}\right) \otimes$ $K\left(X_{D}\right)$ and the summand $K(C \otimes D)^{\oplus 2}$ is mapped into $K\left(X_{\psi} \times X_{D}\right)$ via $f+g$. Therefore, $K(C \otimes D)^{\oplus 2} \rightarrow K\left(X_{\psi} \times X_{D}\right) /\left(K\left(X_{\psi}\right) \otimes K\left(X_{D}\right)\right)$ is an epimorphism. 2. If the algebra $C \otimes D$ is a skewfield, then its class generates the group $K(C \otimes D)$. The images of this class under $f, g$ are $\mathcal{F}, \mathcal{G} \in K\left(X_{\psi}\right) \otimes K\left(X_{D}\right)$.

Corollary 3.5. If $C \otimes D$ is a skewfield, then the group $G^{*} K\left(X_{\psi} \times X_{D}\right)$ is torsion-free.

Proof. By Lemma 3.4, $K\left(X_{\psi} \times X_{D}\right)=K\left(X_{\psi}\right) \otimes K\left(X_{D}\right)$. By Corollary 3.3, the group $G^{*}\left(K\left(X_{\psi}\right) \otimes K\left(X_{D}\right)\right)$ is torsion-free.

\section{The Group Tors $\mathrm{CH}^{2}\left(X_{\psi} \times X_{D}\right)$}

Theorem 4.1. Let $D$ be a biquaternion algebra and $\psi$ be an anisotropic 4dimensional quadratic form with $\operatorname{det} \psi \neq 1$. Then the group $\mathrm{CH}^{2}\left(X_{\psi} \times X_{D}\right)$ is torsion-free except (possibly) the following two cases:

(1) ind $C_{0}(\psi) \otimes D=$ ind $D=4$,

(2) ind $C_{0}(\psi) \otimes D=2$.

Proof. Set $C \stackrel{\text { def }}{=} C_{0}(\psi)$ and $s \stackrel{\text { def }}{=} \operatorname{ind}(C \otimes D)$. The possible values of $s$ are 1,2 , 4, 8.

Assume that $s=8$. Since $\operatorname{det} \psi \neq 1$, it follows that $C \otimes D$ is a skewfield. Therefore, the group $\mathrm{CH}^{2}\left(X_{\psi} \times X_{D}\right) \simeq G^{2} K\left(X_{\psi} \times X_{D}\right)$ is torsion-free by Corollary 3.5. 
Now assume $s=1$. Then $\operatorname{ind}\left(C_{0}\left(\psi_{F(D)}\right)\right)=1$. Therefore the quadratic form $\psi_{F(D)}$ is isotropic. Hence the extension $F(\psi, D) / F(D)$ is purely transcendental and $H^{3}(F(\psi, D) / F)=H^{3}(F(D) / F)$. Now, Corollary 2.2 shows that Tors $\mathrm{CH}^{2}\left(X_{\psi} \times X_{D}\right)=0$.

Finally, assume $s=4$ and ind $D \neq 4$. Then the biquaternion algebra $D$ is Brauer equivalent to a quaternion $F$-algebra $D^{\prime}$. Since $\operatorname{Tors~} \mathrm{CH}^{2}\left(X_{\psi} \times X_{D}\right)$ depends only on the Brauer class of $D$ (see e.g. [8]), we may replace $D$ by $D^{\prime}$. Let $\psi^{\prime}$ be a 3-dimensional quadratic $F$-form such that $C_{0}\left(\psi^{\prime}\right) \simeq D^{\prime}$. The Severi-Brauer variety $X_{D^{\prime}}$ is isomorphic to the conic $X_{\psi^{\prime}}$. Since the tensor product $C_{0}(\psi) \otimes C_{0}\left(\psi^{\prime}\right)$ has index 4 , it is a division algebra. Therefore, by [7, Corollary 4.4], the group $\mathrm{CH}^{2}\left(X_{\psi} \times X_{\psi^{\prime}}\right)$ is torsion-free.

Remark 4.2. The assumption $\psi$ is anisotropic is not essential: if $\psi$ is isotropic, then $H^{3}(F(\psi, D) / F)=H^{3}(F(D) / F)$ and therefore the group $\mathrm{CH}^{2}\left(X_{\psi} \times X_{D}\right)$ is torsion-free as well (by Corollary 2.2).

Proposition 4.3. Let $D$ be a biquaternion algebra and $\psi$ be a 4-dimensional quadratic form with det $\psi \neq 1$. Then the group $\operatorname{Tors~} \mathrm{CH}^{2}\left(X_{\psi} \times X_{D}\right)$ is equal to zero or isomorphic to $\mathbb{Z} / 2$.

Proof. Since we already know that the torsion in the group $\mathrm{CH}^{2}\left(X_{\psi} \times X_{D}\right)$ is annihilated by 2 (Corollary 2.2), it suffices to show that the torsion is cyclic.

Once again we set $C \stackrel{\text { def }}{=} C_{0}(\psi)$. According to Theorem 4.1, it suffices to consider the case where ind $(C \otimes D)$ equals 2 or 4 .

Consider the quotient $K\left(X_{\psi} \times X_{D}\right) /\left(K\left(X_{\psi}\right) \otimes K\left(X_{D}\right)\right)$ with the filtration induced by the topological filtration on $K\left(X_{\psi} \times X_{D}\right)$. Since in the exact sequence of the adjoint graded groups

$$
\begin{aligned}
0 \rightarrow G^{*}\left(K\left(X_{\psi}\right) \otimes K\left(X_{D}\right)\right) & \rightarrow G^{*} K\left(X_{\psi} \times X_{D}\right) \rightarrow \\
& \rightarrow G^{*}\left(K\left(X_{\psi} \times X_{D}\right) /\left(K\left(X_{\psi}\right) \otimes K\left(X_{D}\right)\right)\right) \rightarrow 0
\end{aligned}
$$

the left-hand side term is torsion-free (Corollary 3.3), we have an injection

$$
\text { Tors } G^{*} K\left(X_{\psi} \times X_{D}\right) \hookrightarrow G^{*}\left(K\left(X_{\psi} \times X_{D}\right) /\left(K\left(X_{\psi}\right) \otimes K\left(X_{D}\right)\right)\right) \text {. }
$$

Since $\mathrm{CH}^{2}\left(X_{\psi} \times X_{D}\right) \simeq G^{2} K\left(X_{\psi} \times X_{D}\right)$, it suffices to show that the group $G^{2}\left(K\left(X_{\psi} \times X_{D}\right) /\left(K\left(X_{\psi}\right) \otimes K\left(X_{D}\right)\right)\right)$ is cyclic.

Denote by $h \in K\left(X_{\psi}\right)$ the class of a general hyperplane section of $X_{\psi}$. Let $\xi \in K\left(\bar{X}_{D}\right)$ be the class of the tautological linear bundle on the projective space $\bar{X}_{D}$. Note that for any $i \geq 0$, the multiple (ind $D^{\otimes i}$ ) $\cdot \xi^{i}$ of $\xi^{i}$ belongs to $K\left(X_{D}\right)$. Thus $\xi^{i} \in K\left(X_{D}\right)$ for $i$ even and $4 \xi^{i} \in K\left(X_{D}\right)$ for $i$ odd.

The algebra $C \otimes D$ is simple. Therefore, its Grothendieck group is cyclic. By Lemma 3.4, it follows that the quotient $K\left(X_{\psi} \times X_{D}\right) /\left(K\left(X_{\psi}\right) \otimes K\left(X_{D}\right)\right)$ is generated by two elements, namely by $(s / 2) x$ and $(s / 2) y$, where $x \stackrel{\text { def }}{=}(4+$ $\left.2 h+h^{2}\right) \otimes \xi, y \stackrel{\text { def }}{=}\left(4+2 h+h^{2}\right) \otimes \xi^{3}$, and $s \stackrel{\text { def }}{=}$ ind $C \otimes D$ (we use here the equality $[\mathcal{U}(2)]=4+2 h+h^{2} \in K\left(X_{\psi}\right)$, [12, Lemma 3.6]).

We have a congruence $x \equiv h^{2} \otimes(\xi-1)-h \otimes(\xi-1)^{2}\left(\bmod K\left(X_{\psi}\right) \otimes K\left(X_{D}\right)\right)$. The right side hand element belongs to $K\left(X_{\psi} \times X_{D}\right)^{(2)}$, because it is in $K\left(\bar{X}_{\psi} \times\right.$ 
$\left.\bar{X}_{D}\right)^{(2)}$ and $K\left(X_{\psi} \times X_{D}\right)^{(2)}=K\left(\bar{X}_{\psi} \times \bar{X}_{D}\right)^{(2)} \cap K\left(X_{\psi} \times X_{D}\right)$ (see [26, Lemme 6.3, (i)]). Therefore, $(s / 2) x \in\left(K\left(X_{\psi} \times X_{D}\right) /\left(K\left(X_{\psi}\right) \otimes K\left(X_{D}\right)\right)\right)^{(2)}$ (take into account that the coefficient $s / 2$ is an integer).

We also have another congruence modulo $K\left(X_{\psi}\right) \otimes K\left(X_{D}\right)$ :

$$
y-x \equiv\left(h^{2} \otimes(\xi-1)-h \otimes(\xi-1)^{2}\right) \cdot\left(1 \otimes\left(\xi^{2}-1\right)\right) .
$$

The right side hand element is in $K\left(X_{\psi} \times X_{D}\right)^{3}$ as a product of an element in $K\left(X_{\psi} \times X_{D}\right)^{(2)}$ and the element $1 \otimes\left(\xi^{2}-1\right) \in K\left(X_{\psi} \times X_{D}\right)^{(1)}$. Therefore, $(s / 2)(y-x) \in\left(K\left(X_{\psi} \times X_{D}\right) /\left(K\left(X_{\psi}\right) \otimes K\left(X_{D}\right)\right)\right)^{(3)}$ and it follows that the group $G^{2}\left(K\left(X_{\psi} \times X_{D}\right) /\left(K\left(X_{\psi}\right) \otimes K\left(X_{D}\right)\right)\right)$ is generated by $(s / 2) x$. So, in particular, this group is cyclic.

Remark 4.4. The assumption $\operatorname{det} \psi \neq 1$ is not essential: if $\operatorname{det} \psi=1$, then $H^{3}(F(\psi, D) / F)=H^{3}\left(F\left(\psi^{\prime}, D\right) / F\right)$ and $H^{3}(F(\psi) / F)=H^{3}\left(F\left(\psi^{\prime}\right) / F\right)$, where $\psi^{\prime}$ is an arbitrary 3 -dimensional subform of $\psi$ ([7, Lemma 5.2]). Therefore,

$$
\mathrm{CH}^{2}\left(X_{\psi} \times X_{D}\right) \simeq \mathrm{CH}^{2}\left(X_{\psi^{\prime}} \times X_{D}\right) \simeq \mathrm{CH}^{2}\left(X_{D^{\prime}} \times X_{D}\right)
$$

where $D^{\prime}$ is the even Clifford algebra of $\psi^{\prime}$. The group Tors $\mathrm{CH}^{2}\left(X_{D^{\prime}} \times X_{D}\right)$ is zero or $\mathbb{Z} / 2$ according to [15, Théorème 6.1$]$.

Theorem 4.5. Suppose that $\psi=\langle-x,-y, x y, d\rangle$ (with $d \notin F^{* 2}$ ) and $D=$ $(x, y) \otimes(u, v)$ where $x, y, d, u, v \in F^{*}$. Then Tors $\mathrm{CH}^{2}\left(X_{\psi} \times X_{D}\right)=0$.

Proof. The even Clifford algebra of the quadratic form $\psi$ is isomorphic to the quaternion algebra $(x, y)_{F(\sqrt{d})}$. Therefore, the tensor product $C_{0}(\psi) \otimes D$ is Brauer equivalent to the quaternion algebra $(u, v)_{F(\sqrt{d})}$ and in particular has index 2 or 1 . In the case, where the index is 1, we are done by Theorem 4.1. Let us assume the index equals to 2 . It suffices to show that the element $x \stackrel{\text { def }}{=} h^{2} \otimes(\xi-1)-h \otimes(\xi-1)^{2}$ is in $K\left(X_{\psi} \times X_{D}\right)^{(3)}$ (see the proof of Proposition 4.3).

By definition, the element $h \in K\left(X_{\psi}\right)$ is the class of a hyperplane section of the quadric $X_{\psi}$. This hyperplane section is the quadric $X_{\psi^{\prime}}$ determined by a 3-dimensional subform $\psi^{\prime}$ of $\psi$. Clearly, $x$ is equal to the image of $x^{\prime} \stackrel{\text { def }}{=}$ $h^{\prime} \otimes(\xi-1)-1 \otimes(\xi-1)^{2} \in K\left(X_{\psi^{\prime}} \times X_{D}\right)$ under the push-forward homomorphism $K\left(X_{\psi^{\prime}} \times X_{D}\right) \rightarrow K\left(X_{\psi} \times X_{D}\right)$. Moreover, $x^{\prime} \in K\left(X_{\psi^{\prime}} \times X_{D}\right)^{(2)}$, because $x^{\prime} \in$ $K\left(\bar{X}_{\psi^{\prime}} \times \bar{X}_{D}\right)^{(2)}$ and $K\left(X_{\psi^{\prime}} \times K\left(X_{D}\right)\right)^{(2)}=K\left(\bar{X}_{\psi^{\prime}} \times \bar{X}_{D}\right)^{(2)} \cap K\left(X_{\psi^{\prime}} \times K\left(X_{D}\right)\right)$. Since the codimension of $X_{\psi^{\prime}} \times X_{D}$ in $X_{\psi} \times X_{D}$ equals to 1, it follows that $x \in K\left(X_{\psi} \times X_{D}\right)^{(3)}$.

Remark 4.6. The assumption $d \notin F^{* 2}$ is not essential: if $d \in F^{* 2}$, then Tors $\mathrm{CH}^{2}\left(X_{\psi} \times X_{D}\right) \simeq \operatorname{Tors} \mathrm{CH}^{2}\left(X_{C_{0}\left(\psi^{\prime}\right)} \times X_{D}\right)$, where $\psi^{\prime} \stackrel{\text { def }}{=}\langle-x,-y, x y\rangle$; since $C_{0}\left(\psi^{\prime}\right) \simeq(x, y)$, we have ind $C_{0}\left(\psi^{\prime}\right) \otimes D \leq 2$; therefore, by [15, Théorème $6.1]$, the latter group is zero. 


\section{Standard isotropy in the Case Tors $\mathrm{CH}^{2}\left(X_{\psi} \times X_{D}\right)=0$}

Definition 5.1. We say that $(\phi, D, q)$ is a special triple if the following conditions hold:

1) $\phi$ is an 8-dimensional anisotropic form with $\operatorname{det} \phi=1$,

2) $D$ is a biquaternion algebra,

3) $q$ is an Albert form,

4) $[D]=c(\phi)=c(q) \in \operatorname{Br}_{2}(F)$.

In this section we need the following

Theorem 5.2 ([18, Théorème 4] and [2, Corollary 9.3], see also [8] ). Let $\phi$ be an anisotropic 8-dimensional quadratic form with $\operatorname{det} \phi=1$ and let $D$ be an algebra such that $c(\phi)=[D]$. Then $\phi_{F(D)}$ is anisotropic.

Our study of isotropy of 8-dimensional forms over function field of quadrics is based on the following assertion.

Proposition 5.3. Let $(\phi, D, q)$ be a special triple and $\psi$ be a quadratic form. Then

1. The following two conditions are equivalent:

(i) $\phi+q \in I^{3}(F(\psi, D) / F)$;

(ii) $\phi_{F(\psi)}$ is isotropic.

2. The following two conditions are equivalent:

(i) $\phi+q \in I^{3}(F(\psi) / F)+I^{3}(F(D) / F)+I^{4}(F)$;

(ii) there exists a 5-dimensional Pfister neighbor $\phi_{0}$ such that $\phi_{0} \subset \phi$ and $\left(\phi_{0}\right)_{F(\psi)}$ is isotropic.

Proof. (1i) $\Rightarrow$ (1ii). Condition (1i) implies that the quadratic form $(\phi \perp q)_{F(\psi, D)}$ is hyperbolic. Since $q_{F(D)}$ is hyperbolic, it follows that $\phi_{F(\psi, D)}$ is hyperbolic. Let $E \stackrel{\text { def }}{=} F(\psi)$. We see that $\phi_{E(D)}$ is hyperbolic. Theorem 5.2 implies that $\phi_{E}$ is isotropic, i.e., condition (1ii) holds.

(1ii) $\Rightarrow(1 \mathrm{i})$. Suppose that $\phi_{F(\psi)}$ is isotropic. Since $c(\phi)=c(q)$, it follows that $\phi+q \in I^{3}(F)$. Hence it is sufficient to prove that $\phi_{F(\psi, D)}$ and $q_{F(\psi, D)}$ are hyperbolic. The form $q_{F(\psi, D)}$ is hyperbolic because $q_{F(D)}$ is. Since $c(\phi)=[D]$, we have $\phi_{F(\psi, D)} \in I^{3}(F(\psi, D))$. Since $\phi_{F(\psi)}$ is isotropic, we have $\operatorname{dim}\left(\phi_{F(\psi, D)}\right)_{\text {an }}<$ 8. The Arason-Pfister Hauptsatz shows that $\phi_{F(\psi, D)}$ is hyperbolic.

$(2 \mathrm{i}) \Rightarrow(2 \mathrm{ii})$. By Lemmas 2.3 and 2.4 , there exist $\pi \in P_{3}(F(\psi) / F)$ and $s \in F^{*}$ such that

$$
\phi+q \equiv \pi+q\langle\langle s\rangle\rangle \quad\left(\bmod I^{4}(F)\right) .
$$

We have $\phi+s q \equiv \pi\left(\bmod I^{4}(F)\right)$. Since $\pi \in P_{3}(F), \pi_{F(\pi)}$ is hyperbolic. Hence $(\phi+s q)_{F(\pi)} \equiv \pi_{F(\pi)} \equiv 0\left(\bmod I^{4}(F)\right)$. Since $\operatorname{dim}(\phi+s q) \leq 8+4<16$, the Arason-Pfister Hauptsatz shows that $(\phi+s q)_{F(\pi)}$ is hyperbolic. Hence there exists a form $\gamma$ such that $(\phi \perp s q)_{a n}=\pi \gamma$. Clearly $0<8-6 \leq \operatorname{dim}(\phi \perp$ $s q)_{a n} \leq 8+6<16$. This implies that $\operatorname{dim} \gamma=1$, i.e., there exists $k \in F^{*}$ such that $\gamma=\langle k\rangle$. Thus $\phi+s q=k \pi$. Therefore $(\phi \perp-k \pi)=-s q$. Hence $\phi$ and 
$k \pi$ contain a common subform of dimension

$$
\frac{\operatorname{dim} \phi+\operatorname{dim} \pi-\operatorname{dim} q}{2}=\frac{8+8-6}{2}=5 .
$$

Let us denote such a form by $\phi_{0}$. Since $\operatorname{dim} \phi_{0}=5$ and $\phi_{0} \subset k \pi$, it follows that $\phi_{0}$ is a Pfister neighbor of $\pi$. Since $\pi \in P_{3}(F(\psi) / F)$, it follows that $\left(\phi_{0}\right)_{F(\psi)}$ is isotropic.

$(2 \mathrm{ii}) \Rightarrow(2 \mathrm{i})$. Let $\phi_{0}$ be a 5 -dimensional quadratic form such as in (2ii). By the assumption, there exists $\pi \in G P_{3}(F)$ such that $\phi_{0} \subset \pi$. Since $\left(\phi_{0}\right)_{F(\psi)}$ is isotropic, it follows that $\pi \in G P_{3}(F(\psi) / F)$.

Since $\phi_{0} \subset \phi$ and $\phi_{0} \subset \pi$, there exist 3-dimensional quadratic forms $\rho^{\prime}, \rho^{\prime \prime}$ such that $\phi=\phi_{0} \perp \rho^{\prime}$ and $\pi=\phi_{0} \perp \rho^{\prime \prime}$. We set $\rho=\rho^{\prime \prime} \perp-\rho^{\prime}$. Clearly $\operatorname{dim} \rho=6$. In the Witt ring $W(F)$ we have $\rho=\rho^{\prime \prime}-\rho^{\prime}=\pi-\phi$. In particular, $\rho \in I^{2}(F)$. Hence $\rho$ is an Albert form.

We have $c(\rho)=c(\pi)+c(\phi)=0+c(\phi)=c(q)$. Hence $\rho$ is similar to $q([10$, Theorem 3.12]). Let $s \in F^{*}$ be such that $\rho=s q$. We have $\pi-\phi=\rho=s q$. Hence $\phi=\pi-s q$. Therefore

$$
\begin{aligned}
\phi+q=\pi+q\langle\langle s\rangle\rangle \in G P_{3}(F(\psi) / F) & +[q] \cdot I(F) \subset \\
& \subset I^{3}(F(\psi) / F)+I^{3}(F(D) / F)+I^{4}(F) .
\end{aligned}
$$

Corollary 5.4. Let $(\phi, D, q)$ be a special triple and $\psi$ be a quadratic form of dimension $\geq 3$. Suppose that Tors $\mathrm{CH}^{2}\left(X_{\psi} \times X_{D}\right)=0$. Then the following conditions are equivalent:

(1) $\phi_{F(\psi)}$ is isotropic,

(2) there exits a 5-dimensional Pfister neighbor $\phi_{0}$ such that $\phi_{0} \subset \phi$ and $\left(\phi_{0}\right)_{F(\psi)}$ is isotropic.

Proof. Obvious in view of Proposition 5.3 and Corollary 2.6.

\section{The group $H^{3}(F(\psi, D) / F)$ IN The CASE $\operatorname{ind}\left(C_{0}(\psi) \otimes_{F} D\right)=2$}

In this section we study the group $H^{3}(F(\psi, D) / F)$ in the case where $\psi$ is a 4 dimensional quadratic form with a non-trivial discriminant, $D$ is a biquaternion division $F$-algebra, and ind $\left(C_{0}(\psi) \otimes_{F} D\right)=2$.

Let $d \stackrel{\text { def }}{=} \operatorname{det} \psi$. By our assumption, $d \notin F^{* 2}$. Replacing $\psi$ by a similar form, we can suppose $\psi=\langle-a,-b, a b, d\rangle$ with $a, b \in F^{*}$. Let $L \stackrel{\text { def }}{=} F(\sqrt{d})$. By our assumption, we have $\operatorname{ind}\left((a, b) \otimes_{F} D\right)_{L}=2$. Hence there exists a quaternion $F$-algebra $Q$ such that $(a, b)_{L}+\left[D_{L}\right]=\left[Q_{L}\right]$ in $\operatorname{Br}_{2}(L)$ (see $[16$, Proposition 16.2]). Let us write $Q$ in the form $Q=(r, s)$ with $r, s \in F^{*}$. We set $\psi^{\prime}=\langle-r,-s, r s, d\rangle$.

Let $q$ be an Albert form corresponding to $D$.

Lemma 6.1. There exist $k, k^{\prime} \in F^{*}$ such that $k \psi+k^{\prime} \psi^{\prime}+q \in I^{3}(F)$. 
Proof. Since $(a, b)_{L}+\left[D_{L}\right]=\left[Q_{L}\right]=(r, s)_{L}$, it follows that $(a, b)+(r, s)+[D] \in$ $\operatorname{Br}_{2}(L / F)$. Hence there exist $k \in F^{*}$ such that $(a, b)+(r, s)+[D]=(d, k)$. Let $k^{\prime} \stackrel{\text { def }}{=}-1$ and $\phi \stackrel{\text { def }}{=} k \psi+k^{\prime} \psi^{\prime}+q$. We claim that $\phi \in I^{3}(F)$. To prove this, it is sufficient to verify that $\phi \in I^{2}(F)$ and $c(\phi)=0$. We have

$$
\begin{gathered}
\phi=k \psi+k^{\prime} \psi^{\prime}+q=k\langle-a,-b, a b, d\rangle-\langle-r,-s, r s, d\rangle+q= \\
=k(\langle\langle a, b\rangle\rangle-\langle\langle d\rangle\rangle)-(\langle\langle r, s\rangle\rangle-\langle\langle d\rangle\rangle)+q= \\
=k\langle\langle a, b\rangle\rangle+\langle\langle d, k\rangle\rangle-\langle\langle r, s\rangle\rangle+q .
\end{gathered}
$$

Hence $\phi \in I^{2}(F)$ and $c(\phi)=(a, b)+(d, k)+(r, s)+c(q)=(a, b)+(d, k)+$ $(r, s)+[D]=0$.

Definition 6.2. Let $D$ be a biquaternion algebra and $\psi$ be a 4 -dimensional quadratic form such that $\operatorname{det} \psi \neq 1$ and $\operatorname{ind}\left(C_{0}(\psi) \otimes_{F} D\right)=2$. We denote by $\Gamma(\psi, D)$ the set defined as follows

$\left\{\gamma \in I^{3}(F) \mid\right.$ there exist $k, k^{\prime}, l \in F^{*}$ such that $\left.\gamma=k \psi+k^{\prime} \psi^{\prime}+l q\right\}$,

where $q$ is an Albert form corresponding to $D$ and $\psi^{\prime}$ is a 4-dimensional quadratic form satisfying the following two properties: $\operatorname{det} \psi^{\prime}=\operatorname{det} \psi$ and $C_{0}\left(\psi^{\prime}\right)$ is Brauer-equivalent to $C_{0}(\psi) \otimes_{F} D$.

Remark 6.3. $\quad 1$. The set $\Gamma(\psi, D)$ does not depend on the choice of $q$ and $\psi^{\prime}$ : indeed, the condition on $q$ and $\psi^{\prime}$ determines them uniquely up to similarity.

2. Lemma 6.1 shows the set $\Gamma(\psi, D)$ is not empty.

Lemma 6.4. $\Gamma(\psi, D) \subset I^{3}(F(\psi, D) / F)$.

Proof. Let $\gamma=k \psi+k^{\prime} \psi^{\prime}+l q \in \Gamma(\psi, D)$. By the definition of $\Gamma(\psi, D)$, we have $\gamma \in I^{3}(F)$. Thus it is sufficient to prove that $\gamma_{F(\psi, D)}$ is hyperbolic. We have $\operatorname{dim}\left(\psi_{F(\psi)}\right)_{a n} \leq 2$ and $\operatorname{dim}\left(q_{F(D)}\right)_{a n}=0$. Therefore $\operatorname{dim}\left(\gamma_{F(\psi, D)}\right)_{a n}=$ $\operatorname{dim}\left(\left(k \psi \perp k^{\prime} \psi^{\prime} \perp l q\right)_{F(\psi, D)}\right)_{a n} \leq 2+4+0=6<8$. Since $\gamma \in I^{3}(F)$, the Arason-Pfister Hauptsatz shows that $\gamma_{F(\psi, D)}$ is hyperbolic.

Lemma 6.5. Let $\gamma \in \Gamma(\psi, D), \pi \in P_{3}(F(\psi) / F)$, and $s \in F^{*}$. Then there exists $\gamma^{\prime} \in \Gamma(\psi, D)$ such that $\gamma^{\prime} \equiv \gamma+\pi+q\langle\langle s\rangle\rangle\left(\bmod I^{4}(F)\right)$.

Proof. Let us write $\gamma$ in the form $\gamma=k \psi+k^{\prime} \psi^{\prime}+l q$. Since $\pi \in P_{3}(F(\psi) / F)$, there exists $r \in F^{*}$ such that $\pi=\psi\langle\langle r\rangle\rangle([6$, Lemmas 6.1 and 6.2]).

We have $k \psi+\pi \equiv k \psi-k \pi=k \psi-k \psi\langle\langle r\rangle\rangle=r k \psi\left(\bmod I^{4}(F)\right)$ and $l q+q\langle\langle s\rangle\rangle \equiv l q-l q\langle\langle s\rangle\rangle=l s q\left(\bmod I^{4}(F)\right)$. Therefore

$\gamma+\pi+q\langle\langle s\rangle\rangle=k \psi+k^{\prime} \psi^{\prime}+l q+\pi+q\langle\langle s\rangle\rangle \equiv r k \psi+k^{\prime} \psi^{\prime}+l s q \quad\left(\bmod I^{4}(F)\right)$.

Now it is sufficient to set $\gamma^{\prime}=r k \psi+k^{\prime} \psi^{\prime}+l s q$.

Corollary 6.6. $\Gamma(\psi, D)+I^{4}(F)=\Gamma(\psi, D)+I^{3}(F(\psi) / F)+I^{3}(F(D) / F)+$ $I^{4}(F)$.

Proof. Obvious in view of Lemmas 6.5, 2.3, and 2.4. 
Lemma 6.7. The following conditions are equivalent:

(1) $I^{3}(F(\psi, D) / F) \subset I^{3}(F(\psi) / F)+I^{3}(F(D) / F)+I^{4}(F)$;

(2) $\Gamma(\psi, D) \subset I^{3}(F(\psi) / F)+I^{3}(F(D) / F)+I^{4}(F)$;

(3) there exists $\gamma \in \Gamma(\psi, D)$ such that $\gamma \in I^{3}(F(\psi) / F)+I^{3}(F(D) / F)+I^{4}(F)$;

(4) $\Gamma(\psi, D)$ contains a hyperbolic form, i.e. $0 \in \Gamma(\psi, D)$;

(5) there exist $x, y, u, v, d \in F^{*}$ such that $\psi \sim\langle-x,-y, x y, d\rangle$ and $D \cong$ $(x, y) \otimes_{F}(u, v)$

(6) $\operatorname{Tors} \mathrm{CH}^{2}\left(X_{\psi} \times X_{D}\right)=0$;

(7) $H^{3}(F(\psi, D) / F)=H^{3}(F(\psi) / F)+H^{3}(F(D) / F)$.

Proof. $(1) \Rightarrow(2)$. Obvious in view of Lemma 6.4 .

$(2) \Rightarrow(3)$. Obvious, because $\Gamma(\psi, D)$ is not empty.

$(3) \Rightarrow(4)$. Condition $(3)$ implies that $0 \in \Gamma(\psi, D)+I^{3}(F(\psi) / F)+I^{3}(F(D) / F)+$ $I^{4}(F)$. It follows from Corollary 6.6 that $0 \in \Gamma(\psi, D)+I^{4}(F)$. Hence there exists $\gamma=k \psi+k^{\prime} \psi^{\prime}+l q \in \Gamma(\psi, D)$ such that $\gamma \in I^{4}(F)$. Since $\operatorname{dim} \gamma=$ $4+4+6=14<16$, the Arason-Pfister Hauptsatz shows that $\gamma=0$.

$(4) \Rightarrow(5)$. Let $\gamma=k \psi+k^{\prime} \psi^{\prime}+l q$ be a hyperbolic form. We have $(k \psi \perp l q)_{a n}=$ $-k^{\prime} \psi_{a n}^{\prime}$. Therefore $k \psi$ and $-l q$ contain a common subform of dimension

$$
\frac{1}{2}\left(\operatorname{dim} \psi+\operatorname{dim} q-\operatorname{dim} \psi^{\prime}\right)=\frac{1}{2}(4+6-4)=3 .
$$

Let us denote such a 3-dimensional form by $\tau$. Let $x, y \in F^{*}$ be such that $\tau \sim\langle-x,-y, x y\rangle$. Thus $\langle-x,-y, x y\rangle$ is similar to a subform of $\psi$ and similar to a subform of $q$. Let $d=\operatorname{det} \psi$. Since $\langle-x,-y, x y\rangle$ is similar to a subform of $\psi$ it follows that $\langle-x,-y, x y, d\rangle$ is similar to $\psi$. Since $\langle-x,-y, x y\rangle$ is similar to a subform of the Albert form $q$, it follows that there exist $u, v \in F^{*}$ such that $q$ is similar to $\langle-x,-y, x y, u, v,-u v\rangle$. Then $[D]=c(q)=(x, y)+(u, v)$. Therefore $D \cong(x, y) \otimes_{F}(u, v)$.

$(5) \Rightarrow(6)$. See Theorem 4.5.

$(6) \Rightarrow(7)$. See Proposition 2.1.

$(7) \Rightarrow(1)$. See Lemma 2.5.

Proposition 6.8. Let $D$ be a biquaternion algebra and $\psi$ be a 4-dimensional quadratic form such that $\operatorname{det} \psi \neq 1$ and $\operatorname{ind}\left(D \otimes_{F} C_{0}(\psi)\right)=2$. Then for any $\gamma \in \Gamma(\psi, D)$ one has

$$
H^{3}(F(\psi, D) / F)=H^{3}(F(\psi) / F)+H^{3}(F(D) / F)+e^{3}(\gamma) H^{0}(F) .
$$

Proof. By Lemma 6.4, the element $e^{3}(\gamma)$ belongs to $H^{3}(F(\psi, D) / F)$. If the group $\mathrm{CH}^{2}\left(X_{\psi} \times X_{D}\right)$ is torsion-free, then, by Proposition 2.1, we have

$$
H^{3}(F(\psi, D) / F)=H^{3}(F(\psi) / F)+H^{3}(F(D) / F)
$$

and the proof is complete. If Tors $\mathrm{CH}^{2}\left(X_{\psi} \times X_{D}\right) \neq 0$, Lemma 6.7 shows that $\gamma \notin I^{3}(F(\psi) / F)+I^{3}(F(D) / F)+I^{4}(F)$. It follows from Lemma 2.5 that $e^{3}(\gamma) \notin H^{3}(F(\psi) / F)+H^{3}(F(D) / F)$. To complete the proof it is sufficient to apply Proposition 4.3 and Proposition 2.1. 
Corollary 6.9. $I^{3}(F(\psi, D) / F) \subset I^{3}(F(\psi) / F)+I^{3}(F(D) / F)+\Gamma^{\prime}(\psi, D)+$ $I^{4}(F)$, where $\Gamma^{\prime}(\psi, D)=\Gamma(\psi, D) \cup\{0\}$.

Proof. Let $\tau \in I^{3}(F(\psi, D) / F)$. Choose an element $\gamma \in \Gamma(\psi, D)$. By Proposition 6.8, either the element $e^{3}(\tau)$ or the element $e^{3}(\tau-\gamma)$ is in $H^{3}(F(\psi) / F)+$ $H^{3}(F(D) / F)$. It remains to apply Lemma 2.5 .

Proposition 6.10. Let $\lambda \in I^{3}(F(\psi, D) / F)$. Then at least one of the following conditions holds

1) $\lambda \in I^{3}(F(\psi) / F)+I^{3}(F(D) / F)+I^{4}(F)$;

2) $\lambda \in \Gamma(\psi, D)+I^{4}(F)$.

Proof. Obvious in view of Corollaries 6.9 and 6.6.

\section{MAIN THEOREM}

Theorem 7.1. Let $\phi$ be an anisotropic 8-dimensional quadratic form with $\operatorname{det} \phi=1$ and let $\psi$ be a 4-dimensional quadratic form with $\operatorname{det} \psi \neq 1$. Suppose that $\phi_{F(\psi)}$ is isotropic. Then the isotropy of $\phi_{F(\psi)}$ is L-standard except possibly the case ind $C(\phi)=\operatorname{ind}\left(C(\phi) \otimes_{F} C_{0}(\psi)\right)=4$.

Proof. In the case where ind $C(\phi)=8$, the theorem is proved in [6]. Thus we can suppose that ind $C(\phi) \leq 4$. Then there exists a biquaternion algebra $D$ such that $c(\phi)=[D]$. Let $q$ be an Albert form corresponding to $D$. Clearly $(\phi, D, q)$ is a special triple .

By Corollary 5.4, we can suppose that Tors $\mathrm{CH}^{2}\left(X_{\psi} \times X_{D}\right) \neq 0$. Theorem 4.1 asserts that at least one of the following conditions holds:

1) $\operatorname{ind}\left(D \otimes C_{0}(\psi)\right)=$ ind $D=4$,

2) ind $\left(D \otimes C_{0}(\psi)\right)=2$.

If ind $\left(D \otimes C_{0}(\psi)\right)=2$, then all the conditions of Definition 6.2 hold. Thus we have a well-defined set $\Gamma(\psi, D)$.

By Proposition 5.3, we have $\phi+q \in I^{3}(F(\psi, D) / F)$. By Proposition 6.10, we see that at least one of the following condition holds:

1) $\phi+q \in I^{3}(F(\psi) / F)+I^{3}(F(D) / F)+I^{4}(F)$;

2) $\phi+q \in \Gamma(\psi, D)+I^{4}(F)$.

In the first case, Proposition 5.3 shows that there exists a 5-dimensional Pfister neighbor $\phi_{0}$ such that $\phi_{0} \subset \phi$ and $\left(\phi_{0}\right)_{F(\psi)}$ is isotropic. This implies that the isotropy of $\phi_{F(\psi)}$ is L-standard.

Therefore, we may assume that $\phi+q \in \Gamma(\psi, D)+I^{4}(F)$. Thus there exist $k, k^{\prime}, l \in F^{*}$ (and a 4-dimensional quadratic form $\psi^{\prime}$ ) such that

$$
\phi+q \equiv k \psi+k^{\prime} \psi^{\prime}+l q \quad\left(\bmod I^{4}(F)\right) .
$$

Since $k \psi+k^{\prime} \psi^{\prime}+l q \in I^{3}(F)$ it follows that

$$
k \psi+k^{\prime} \psi^{\prime}+l q \equiv l\left(k \psi+k^{\prime} \psi^{\prime}+l q\right) \quad\left(\bmod I^{4}(F)\right) \text {. }
$$

Therefore

$$
\phi+q \equiv l k \psi+l k^{\prime} \psi^{\prime}+q \quad\left(\bmod I^{4}(F)\right) .
$$


Thus $\phi \equiv l k \psi+l k^{\prime} \psi^{\prime}\left(\bmod I^{4}(F)\right)$. Let $\phi^{*} \stackrel{\text { def }}{=} \psi \perp k k^{\prime} \psi^{\prime}$. Since $\phi \equiv l k \phi^{*}$ $\left(\bmod I^{4}(F)\right)$ and $\operatorname{dim} \phi^{*}=8$, it follows that $\phi$ and $\phi^{*}$ are half-neighbors. Since $\psi \subset \phi^{*}$, the isotropy is L-standard and the proof is complete.

Corollary 7.2. Let $\phi$ be an anisotropic 8-dimensional quadratic form with $\operatorname{det} \phi=1$ and let $\psi$ be a 4-dimensional quadratic form with $\operatorname{det} \psi \neq 1$. Suppose that $\phi_{F(\psi)}$ is isotropic but the isotropy is not L-standard. Then $\phi$ can be written in the form $\phi=\pi_{1} \perp \pi_{2}$ with $\pi_{1}, \pi_{2} \in G P_{2}(F)$.

Proof. Since $\operatorname{det} \phi=1$, it is sufficient to verify that $\phi$ contains a 4-dimensional quadratic form with trivial determinant. Suppose at the moment that $\phi$ contains no 4-dimensional quadratic form with trivial determinant. Then [5, Theorem 6.1] implies that there exists a homomorphism of $F$-algebras, $C_{0}(\psi) \rightarrow C_{0}(\phi)$. Since $\operatorname{det} \phi=1$ and $\operatorname{dim} \phi=8$, there exists a 3 -quaternion algebra $A$ such that $C_{0}(\phi)$ has the form $A \times A$ and $C(\phi) \simeq M_{2}(A)$. Thus we get a homomorphism $C_{0}(\psi) \rightarrow A$ which is injective because $C_{0}(\psi)$ is a simple algebra. Then $\operatorname{ind}\left(C_{0}(\psi) \otimes_{F} A\right)=2$. Since $M_{2}(A) \simeq C(\phi)$, we have $\operatorname{ind}\left(C_{0}(\psi) \otimes_{F} C(\phi)\right)=2$. Theorem 7.1 implies that isotropy of $\phi_{F(\psi)}$ is Lstandard. The contradiction obtained completes the proof.

It is a natural question if there exists an example of non-L-standard isotropy. Some way to construct non L-standard isotropy is based on the following

Lemma 7.3. Let $q$ be a 6-dimensional quadratic form and let $\psi$ be a 4-dimensional quadratic form over a field $k$. Suppose that $q$ is a $k(\psi)$-minimal form (see definition in [3]). Let $F \stackrel{\text { def }}{=} k((t)), \phi \stackrel{\text { def }}{=} q \perp t\langle 1, \operatorname{det}(q)\rangle$. Then the form $\phi_{F(\psi)}$ is isotropic, but the isotropy is not L-standard.

Proof. The proof of this lemma is absolutely analogous to the proof of $[6$, Theorem 5.1] and we omit it.

Corollary 7.4. There exist a field $F$, an 8-dimensional quadratic form $\phi \in$ $I^{2}(F)$, and a 4-dimensional $F$-form $\psi$ with nontrivial determinant such that $\phi_{F(\psi)}$ is isotropic, but the isotropy is not L-standard.

Proof. By [9] there exists a field $k$, a 6-dimensional quadratic form $q$ and a 4-dimensional quadratic form $\psi$ over a field $k$ such that $q$ is a $k(\psi)$-minimal form. Now the required result follows from Lemma 7.3.

\section{ISOTROPY OVER THE FUNCTION FIELD OF A CONIC}

In this section we still assume that $\phi$ is an anisotropic 8-dimensional quadratic form of trivial determinant. We are interested in the question when $\phi$ is isotropic over the function field of a quadratic form $\psi$.

For the forms $\psi$ of dimension $\geq 5$, the question was studied in [17] and [18]. The case $\operatorname{dim} \psi=4, d_{ \pm} \psi \neq 1$ is done in the previous section. Thus it suffices to consider only two cases: $\operatorname{dim} \psi=3$ or $\psi \in G P_{2}(F)$. Since the function field of a form $\psi \in G P_{2}(F)$ is stably birational equivalent to the function field of 
an arbitrary 3-dimensional subform of $\psi$ (see e.g. [7, Lemma 5.2]), it suffices to handle only one of these cases.

Let us consider the case where $\psi \in G P_{2}(F)$.

Theorem 8.1. Let $\phi$ be an anisotropic 8-dimensional quadratic form (we do not assume $\operatorname{det} \phi=1)$ and let $\psi \in G P_{2}(F)$. Then the form $\phi_{F(\psi)}$ is isotropic if and only if at least one of the following conditions holds:

a) there exists a 10-dimensional form $\phi^{*}$ such that $\psi \subset \phi^{*}$ and $s \phi \equiv \phi^{*}$ $\left(\bmod I^{4}(F)\right)$ for suitable $s \in F^{*}$;

b) there exists a 5-dimensional subform $\phi_{0} \subset \phi$ with the following two properties:

- the form $\phi_{0}$ is a Pfister neighbor,

- the form $\left(\phi_{0}\right)_{F(\psi)}$ is isotropic.

Proof. First, suppose that $\phi_{F(\psi)}$ is isotropic. The excellence property of the field extension $F(\psi) / F$ implies that there exist a 6-dimensional form $\tau$ and a form $\gamma$ such that $\phi-\tau=\gamma \psi$ (see [3, Proof of Proposition 1.1]). Comparing dimensions, one can see that $1 \leq \operatorname{dim} \gamma \leq 3$. First, consider the case where $\operatorname{dim} \gamma$ is odd (i.e., $\operatorname{dim} \gamma=1$ or 3 ). We set $s \stackrel{\text { def }}{=} d_{ \pm} \gamma$. Clearly, $\gamma \equiv\langle s\rangle$ $\left(\bmod I^{2}(F)\right)$. Hence $\gamma \psi \equiv s \psi\left(\bmod I^{4}(F)\right)$. Since $\phi-\tau=\gamma \psi$, it follows that $\phi=\tau+\gamma \psi \equiv \tau+s \cdot \psi \equiv s(s \tau+\psi)\left(\bmod I^{4}(F)\right)$. Setting $\phi^{*} \stackrel{\text { def }}{=} s \tau \perp \psi$, one can see that condition a) holds. Now, suppose that $\operatorname{dim} \gamma$ is odd, i.e., $\operatorname{dim} \gamma=2$. Then $\gamma \psi \in G P_{3}(F)$. We have $\phi-\gamma \psi=\tau$. Therefore, the quadratic forms $\phi$ and $\gamma \psi$ contain a common subform of dimension $\frac{1}{2}(\operatorname{dim} \phi+\operatorname{dim} \gamma \psi-\operatorname{dim} \tau)=$ $\frac{1}{2}(8+8-6)=5$. Let us denote that 5 -dimensional form by $\phi_{0}$. Clearly, condition b) holds.

Now, suppose that at least one of conditions a) and b) holds. We have to verify that $\phi_{F(\psi)}$ is isotropic. It is obvious in the case where condition b) holds. Suppose now that condition a) holds. Since $\psi \subset \phi^{*}$ and $\operatorname{dim} \phi^{*}=10$, one has $\operatorname{dim}\left(\phi_{F(\psi)}^{*}\right)_{a n} \leq 6$. Therefore $\operatorname{dim}\left(\left(\phi \perp-s \phi^{*}\right)_{F(\psi)}\right)_{a n} \leq 8+6=$ $14<16$. Since $\phi \perp-s \phi^{*} \in I^{4}(F)$, the Arason-Pfister Hauptsatz implies that $\operatorname{dim}\left(\left(\phi \perp-s \phi^{*}\right)_{F(\psi)}\right)_{a n}$ is hyperbolic. Hence $\left(\phi_{F(\psi)}\right)_{a n}=\left(s \phi_{F(\psi)}^{*}\right)_{a n}$. Therefore $\operatorname{dim}\left(\phi_{F(\psi)}\right)_{a n} \leq 6$. Hence $\phi_{F(\psi)}$ is isotropic.

Corollary 8.2. Let $\phi$ be an anisotropic 8-dimensional quadratic form with $\operatorname{det} \phi=1$ and let $\psi$ be a 4-dimensional quadratic form with $\operatorname{det} \psi=1$ (or $\psi$ is a 3-dimensional form). Suppose that $\phi_{F(\psi)}$ is isotropic but the isotropy is not L-standard. Then ind $\left(C(\phi) \otimes C_{0}(\psi)\right)=4$.

Proof. We can assume that $\psi \in G P_{2}(F)$ (if $\operatorname{dim} \psi=3$, then we replace $\psi$ by $\psi \perp\langle\operatorname{det} \psi\rangle)$. Let us suppose that ind $\left(C(\phi) \otimes C_{0}(\psi)\right) \neq 4$. By Theorem 8.1, there exist a 10-dimensional form $\phi^{*}$ and an coefficient $s \in F^{*}$ such that $\psi \subset \phi^{*}$ and $s \phi \equiv \phi^{*}\left(\bmod I^{4}(F)\right)$. Then $\phi^{*}$ can be written in the form $\phi^{*}=\psi \perp q$. Clearly, $q$ is an Albert form and $c(q)=c\left(\phi^{*}\right)+c(\psi)$. Therefore ind $C(q)=\operatorname{ind}\left(C\left(\phi^{*}\right) \otimes C(\psi)\right)=\operatorname{ind}\left(C(\phi) \otimes C_{0}(\psi)\right) \neq 4$. Hence, $q$ is isotropic. Thus there exists a 4-dimensional form $\tilde{q}$ such that $\tilde{q}_{a n}=q_{a n}$. Set $\tilde{\phi}^{*} \stackrel{\text { def }}{=} \psi \perp \tilde{q}$. 
Clearly, $\operatorname{dim} \tilde{\phi}^{*}=8, \psi \subset \tilde{\phi}^{*}$ and $s \phi \equiv \phi^{*} \equiv \tilde{\phi}^{*}\left(\bmod I^{4}(F)\right)$. Therefore, the isotropy $\phi_{F(\psi)}$ is L-standard, a contradiction.

Remark 8.3. There are many examples of $\phi$ and $\psi$ with $\psi \in G P_{2}(F)$ such that the isotropy of $\phi_{F(\psi)}$ is not L-standard. The condition of Theorem 8.1 can be regarded as a modification of the notion of the L-standard isotropy for the case $\psi \in G P_{2}(F)$.

We do not know the answer to the following

Question 8.4. Let $\phi$ be a 8-dimensional quadratic form with trivial determinant and $\psi$ be a 3-dimensional quadratic form such that $\phi_{F(\psi)}$ is isotropic. Is it always true that the isotropy is L-standard?

The answer is evidently positive in the case where ind $C(\phi)=1$. It is not difficult to show that the answer is also positive in the case where ind $C(\phi)=2$.

\section{REFERENCES}

[1] Arason, J. Kr. Cohomologische Invarianten quadratischer Formen. J. Algebra 36 (1975), 448-491.

[2] Esnault, H., Kahn, B., Levine, M., Viehweg, V. The Arason invariant and mod 2 algebraic cycles. $K$-theory Preprint Archives (http://www.math.uiuc.edu/K-theory/), Preprint $\mathrm{N}^{\circ} 151$.

[3] Hoffmann, D. W., Lewis, D. W., van Geel, J. Minimal forms for function fields of conics. Proc. Symp. Pure Math. 58.2 (1995), 227-237.

[4] Izhboldin, O. T. On the nonexcellence of the function fields of Severi-Brauer varieties. Max-Planck-Institut für Mathematik in Bonn, Preprint MPI 96-159 (1996), 1-28.

[5] Izhboldin, O. T. Isotropy of low dimensional forms over the function fields of quadrics. Max-Planck-Institut für Mathematik in Bonn, Preprint MPI 97-1 (1997), 1-18.

[6] Izhboldin O. T., Karpenko N. A. Isotropy of virtual Albert forms over function fields of quadrics. Prépublications de l'Équipe de Mathématique de Besançon 97/07 (1997), 1-11. Revised version: http://www.uni-muenster.de/math/u/scharlau/publ

[7] Izhboldin O. T., Karpenko N. A. Isotropy of 6-dimensional quadratic forms over function fields of quadrics. Prépublications de l'Équipe de Mathématique de Besançon 97/12 (1997), 1-25.

[8] Izhboldin, O. T., Karpenko, N. A. On the group $H^{3}(F(\psi, D) / F)$. Preprint, 1997.

[9] Izhboldin, O. T., Karpenko, N. A. Some new examples in the theory of quadratic forms. Preprint, 1997.

[10] Jacobson, N. Some applications of Jordan norms to involutorial associative algebras. Advances in Math. 48 (1983), 149-165.

[11] Kahn, B. Descente galoisienne et $K_{2}$ des corps de nombres. K-theory 7 (1993), 55-100.

[12] Karpenko, N. A. Algebro-geometric invariants of quadratic forms. Algebra i Analiz 2 (1991), no. 1, 141-162 (in Russian). Engl. transl.: Leningrad (St. Petersburg) Math. J. 2 (1991), no. 1, 119-138.

[13] Karpenko, N. A. On topological filtration for Severi-Brauer varieties II. Amer. Math. Soc. Transl. 174 (1996), no. 2, 45-48.

[14] Karpenko, N. A. Codimension 2 cycles on Severi-Brauer varieties. Prépublications de l'Équipe de Mathémathiques de Besançon 96/40 (1996), 1-26. To appear in K-theory.

[15] Karpenko, N. A. Cycles de codimension 2 en produits de variétés de Severi-Brauer. Publications Mathématiques de la Faculté des Sciences de Besançon — Théorie des Nombres, Années 1994/95-1995/96, 1-15. 
[16] Knus, M.-A., Merkurjev, A. S., Rost, M., Tignol, J.-P. The Book of Involutions, in preparation, preliminary version of June 12, 1996.

[17] Laghribi, A. Formes quadratiques en 8 variables dont l'algèbre de Clifford est d'indice 8. $K$-Theory, to appear.

[18] Laghribi, A. Isotropie de certaines formes quadratiques de dimension 7 et 8 sur le corps des fonctions d'une quadrique. Duke Math. J. 85 (1996), no. 2, 397-410.

[19] Lam, T. Y. The Algebraic Theory of Quadratic Forms. Massachusetts: Benjamin 1973 (revised printing: 1980).

[20] Merkurjev, A. S. On the norm residue symbol of degree 2. Dokl. Akad. Nauk SSSR 261 (1981), 542-547 (in Russian). Engl. transl.: Soviet Math. Dokl. 24 (1981), 546-551.

[21] Merkurjev, A. S., Suslin, A. A. The group $K_{3}$ for a field. Izv. Akad. Nauk SSSR Ser. Mat. 54 (1990), no.3, 522-545 (in Russian). Engl. transl.: Math. USSR, Izv. 36 (1991), no.3, 541-565.

[22] Panin, I. A. On the algebraic K-theory of twisted flag varieties. K-theory 8 (1994), no. $6,541-585$.

[23] Peyre, E. Products of Severi-Brauer varieties and Galois cohomology. Proc. Symp. Pure Math. 58.2 (1995), 369-401.

[24] Quillen, D. Higher algebraic K-theory: I. Lect. Notes Math. 341 (1973), 85-147.

[25] Rost, M. Hilbert 90 for $K_{3}$ for degree-two extensions. Preprint (1986).

[26] Sansuc, J.-J. Groupe de Brauer et arithmétique des groupes algébriques linéaires sur un corps de nombres. J. reine angew. Math. 327 (1981), 12-80.

[27] Scharlau, W. Quadratic and Hermitian Forms Springer, Berlin, Heidelberg, New York, Tokyo (1985).

[28] Suslin, A. A. Algebraic K-theory and the norm residue homomorphism. J. Soviet Math. 30 (1985), 2556-2611.

[29] Swan, R. K-theory of quadric hypersurfaces. Ann. Math. 122 (1985), no. 1, 113-154.

Oleg Izhboldin, Department of Mathematics and Mechanics, St.-Petersburg State University, Petrodvorets, 198904, RUSSiA

E-mail address: oleg@izh.urs.pu.ru

Nikita Karpenko, Westfälische Wilhelms-Universität, Mathematisches InStitut, Einsteinstrasse 62, D-48149 Münster, GERMANY

E-mail address: karpenk@math.uni-muenster.de 